\title{
Kriya Yoga in Patients with Depressive Disorders: A Pilot Study
}

\author{
Ananya Srivastava ${ }^{1}$ Pooja P. Kuppili² Tanu Gupta ${ }^{3}$ \\ ${ }^{1}$ Department of Intern, All India Institute of Medical Sciences, \\ Jodhpur, Rajasthan, India \\ 2 Penn Hospital Black Country Healthcare NHS Foundation Trust, \\ United Kingdom \\ ${ }^{3}$ Department of Psychiatry, All India Institute of Medical Sciences, \\ Jodhpur, Rajasthan, India \\ ${ }^{4}$ Department of Psychiatry, All India Institute of Medical \\ Sciences, Jodhpur, Rajasthan, India \\ ${ }^{5}$ Indra Yog Sansthan, Jodhpur, Rajasthan, India
}

Naresh Nebhinani ${ }^{4} \quad$ Ambika Chandani ${ }^{5}$

\begin{abstract}
Address for correspondence Naresh Nebhinani, Department of Psychiatry, MD, DNB, Diploma in Naturopathy \& Yoga, MNAMS, FAIMER Fellow (GSMC-2014), All India Institute of Medical Science, Jodhpur, Rajasthan 342005, India (e-mail: drnaresh_pgi@yahoo.com).
\end{abstract}

J Neurosci Rural Pract 2021;12:362-367.

\begin{abstract}
Background and Objectives Despite the easy acceptability and holistic nature of Kriya yoga, there are no studies evaluating the role of Kriya yoga intervention on depression. The objective of the current study was to assess the feasibility and effect of adjunctive Kriya yoga on depression.

Methods Patients with major depressive disorder who opted for Kriya yoga were recruited into the intervention group (adjunctive Kriya yoga) and those on psychotro-

Keywords

- alternative medicine

- adjunctive

- Kriya yoga

- major depressive disorder pic medication alone were enrolled into the control group. The Hamilton Depression Rating Scale (HDRS) measurements were recorded at baseline, end of 2, 4, and 8 weeks. Results HDRS scores of the intervention group $(n=29)$ were found to be significantly lesser than that of the control group $(n=52)$ by the end of 2,4 , and 8 weeks. The remission rate was also significantly greater in the intervention group.

Conclusion Kriya yoga intervention was found to be feasible, as well as improved the severity of depression.
\end{abstract}

\section{Key Messages}

- Kriya yoga is a feasible adjunctive therapy in management of patients with major depressive disorder.

- Adjunctive Kriya yoga can improve the symptom severity in subjects with major depressive disorder, in comparison to pharmacological therapy alone.

- Adjunctive Kriya yoga can improve the rate of remission in those suffering with major depressive disorder, in comparison to pharmacological therapy alone.

DOI https://doi.org/

$10.1055 / \mathrm{s}-0041-1726618$

ISSN 0976-3147

\section{Introduction}

Major depressive disorder is a common psychiatric disorder affecting people of all strata and the second leading cause of disability with $20 \%$ lifetime prevalence. ${ }^{1}$ Effectiveness studies suggest low remission rates of 11 to $30 \%$, with antidepressants, which is not considered optimal. ${ }^{2}$ Several studies have demonstrated the benefits of adjunctive yoga in the management of depression. There is growing evidence about the positive effects of the yogic intervention on depression.,

(c)2021. Association for Helping Neurosurgical Sick People.

This is an open access article published by Thieme under the terms of the Creative Commons Attribution-NonDerivative-NonCommercial-License, permitting copying and reproduction so long as the original work is given appropriate credit. Contents may not be used for commercial purposes, or adapted, remixed, transformed or built upon. (https://creativecommons.org/licenses/by-nc-nd/4.0/).

Thieme Medical and Scientific Publishers Pvt. Ltd. A-12, 2nd Floor, Sector 2, Noida-201301 UP, India 
Numerous meta-analyses exist about the efficacy of yoga and mindfulness-based meditation in depression. A meta-analysis performed on 12 randomized controlled trials (RCTs) reported moderate effect size for the short-term effect of yoga. ${ }^{5}$ Another meta-analyses of 39 studies found similar findings of moderate effect size in control of depressive symptoms. ${ }^{6}$ A meta-analysis of 10 RCTs reported moderate-to-large effect sizes of adjunctive yoga intervention in treatment of major depression. ${ }^{7}$

Though yoga has been found to be beneficial in patient with depressive symptoms, there have been varying reports on the effectiveness of adjunctive yoga compared with medication. ${ }^{8-11}$ Furthermore, several gaps in the literature exist such as low sample size, poorly described methodology, high risk of bias, and poor reporting of safety of the intervention.

Patanjali's Kriya yoga, also called "Apas-Swadhyaya-Ishwar Pranidhana," consists of several relaxation exercise, that is, breathing exercises, pranayama, chanting, mudra, shavasan, etc., and brings various psychophysiological changes leading to the total control of the body. ${ }^{12}$ Recitation of "Om" gives rise to sensation, feelings, and experiences of positive nature, which makes the individual want to continue them. ${ }^{13}$ Considering the increasing prevalence of depression and limited rates of remission, Kriya yoga can be an intervention of choice based on its holistic nature, easy availability, and acceptability. Despite these advantages, there have been no studies evaluating the effectiveness of Patanjali's Kriya yoga on depression.

The aim of the current study was to assess the feasibility and effect of Kriya yoga in patients with depressive disorder. The primary objective was to demonstrate the feasibility of Kriya yoga and to compare the symptom severity of depression in those performing Kriya yoga along with psychotropic medication with those receiving psychotropic medication alone.

\section{Materials and Methods}

The study approval was taken from Institutional Ethics Committee. The informed consent of each participant was taken before starting the study.

It was a prospective study performed at the Psychiatry Outpatient setting over 1 year. The patients with depressive disorder were recruited by purposive sampling. The inclusion criteria were patients of either gender aged between 18 to 50 years with a diagnosis of major depressive disorder as per the International Classification of Disease-10th Revision (ICD-10) criteria (as diagnosed by a qualified psychiatrist), who did not have any previous exposure to yoga therapy and who were willing to provide consent to participate in the study. Patients with comorbid psychosis, any other psychiatric diagnosis including substance abuse, mental retardation, comorbid chronic medical disorders, or having sensory or physical impairment interfering with assessment and yoga practices were excluded from the study. The sample size was not calculated a priori as it was a pilot study.
The study patients were divided into two groups. At the beginning of the study, the study patients were offered the choice of adjunctive Kriya yoga. Those who opted for it were recruited into the intervention group (psychotropic medications with Kriya yoga), and those who opted out against Kriya yoga were put under the control group (only psychotropic medications).

\section{Intervention}

Kriya yoga ${ }^{12}$ was developed by a professional yoga trainer which included the following components:

- Complete breath.

- "Anulom-Vilom" (also called alternate nostril breathing, "nadishodan pranayama”).

- "Gayatri Mantra."

- "Om" chanting.

"Aana Pana" (mindfulness-based meditation) and shavasan were added to Kriya yoga to provide the benefit of mindfulness and improve relaxation and concentration. The intervention was validated by yoga trainers with discussion with psychiatrists and psychologist in view of potential benefits, feasibility, and other logistic issues.

The first week of the intervention consisted of supervised 45-minute session daily for 2 weeks at the designated yoga center under the direct supervision of yoga trainer and expert in Kriya yoga. Twenty minutes of practice at home daily was advised throughout the study (8 weeks). Patient compliance was ensured through phone calls. For ease and better compliance, an audio version of Kriya yoga was given to participants for home practice. Feedback was taken from the patients regarding the ease of performing asanas by themselves.

\section{Instruments}

The patients in both the groups were assessed on the following instruments at baseline, 2, 4, and 8 weeks by an independent psychiatrist.

Semistructured proforma was used to record sociodemographic variables and clinical parameters like the age of onset of the illness, duration of depression, time spent in episodes, and current treatment details.

\section{Hamilton Depression Rating Scale}

The Hamilton Depression Rating Scale (HDRS) is a 17-item scale which is rated by the clinician. Each item is rated from 0 to 4 , with ratings of mild (8-13), moderate (14-18), and severe (19 or greater). ${ }^{14}$

\section{Statistical Analysis}

The statistical analysis was done by using the SPSS (Statistical Package for the Social Sciences) version 15.0. ${ }^{15}$ Descriptive statistics have been used to calculate frequencies, percentages, mean, and standard deviation. Comparison between the groups have been done using Chi-square test and $t$-tests for categorical and continuous variables, respectively. For continuous and categorical variables with nonparametric 
distribution, the Mann-Whitney $U$-test and Fisher's exact test were used, respectively.

\section{Results}

Initially, 34 cases in the intervention group and 47 controls were recruited. After initial recruitment, five cases in the intervention group had to be shifted to the control group as they expressed their inability to attend intervention due to logistic reasons. A total of 29 cases in the intervention group and 52 controls were finally recruited in the study.

\section{Demographic and Clinical Profile}

Sociodemographic variables and the clinical profile of the yoga group and the control group, are depicted in - Table $\mathbf{1}$. There was no significant difference with regard to the age of both groups. However, the intervention group had received significantly longer years of education.

The intervention and control groups comprised predominantly of students and housewives, respectively. Both the groups were comparable for gender. The intervention group predominantly belonged to the urban region, and controls predominantly belonged to the rural background.
The majority of the patients in intervention, as well as a control group, suffered from a single episode of moderate depression. No significant difference was noted in the grade of depression across both the groups as per HDRS. All the patients were treated with escitalopram in the dose range of 10 to $20 \mathrm{mg}$.

\section{Intervention Details}

Though initially, we decided for 2 weeks of supervised Kriya yoga sessions, but due to logistics, patients' feasibility, and naturalistic design for our study, they were allowed with a flexible schedule. The mean duration of patients receiving guided intervention was 7.83 (standard deviation [SD] = 4.96) days. The median duration of guided intervention was 7 days. Four cases had received only 1 day of the guided intervention. Nine cases had completed 14 days of guided intervention.

After baseline assessment, we have asked patients in both the groups to follow-up at second, fourth, and eighth weeks.

Overall, 18 patients in the Kriya intervention group and 22 patients in the treatment as usual group had come for follow-up at 2 weeks. Twelve patients in the yoga group and 21 patients in the treatment as usual group had attended

Table 1 Demographic and clinical profile of cases and controls

\begin{tabular}{|c|c|c|c|}
\hline \multirow[t]{2}{*}{ Variable } & $\begin{array}{l}\text { Intervention group } \\
(n=29)\end{array}$ & $\begin{array}{l}\text { Control group } \\
(n=52)\end{array}$ & \multirow{2}{*}{$\begin{array}{l}\text { Statistics } \\
t \text { (p-Value) }\end{array}$} \\
\hline & \multicolumn{2}{|c|}{ Mean (SD)/median (IQR) } & \\
\hline Age (y) & $30.48(10.22)$ & $33.61(8.97)$ & $t=-1.38(p=0.174)$ \\
\hline Number of years of education & $14.59(4.00)$ & $10.15(5.70)$ & $t=4.08(p<0.001)$ \\
\hline Duration of illness in weeks (median) & $16(28)$ & $16(32)$ & $U=686.5(p=0.594)$ \\
\hline \multirow[t]{2}{*}{ Depression severity at baseline (mean HDRS score) } & $22.86(5.83)$ & $23.60(5.70)$ & $t=-0.55(p=0.586)$ \\
\hline & \multicolumn{2}{|l|}{ Frequency (\%) } & \\
\hline \multicolumn{3}{|l|}{ Sex } & \multirow[t]{3}{*}{$\chi^{2}=0.231(p=0.631)$} \\
\hline Male & $15(51.7)$ & $24(46.2)$ & \\
\hline Female & $14(48.3)$ & $28(53.8)$ & \\
\hline \multicolumn{3}{|l|}{ Occupation } & \multirow[t]{3}{*}{$\chi^{2}=1.144(p=0.285)$} \\
\hline Employed & $29(100.0)$ & $50(96.15)$ & \\
\hline Unemployed & $0(0.00)$ & $2(3.85)$ & \\
\hline \multicolumn{3}{|l|}{ Religion } & \multirow[t]{3}{*}{$\chi^{2}=0.041(p=0.840)$} \\
\hline Hindu & $27(93.1)$ & $49(94.2)$ & \\
\hline Muslim & $02(6.9)$ & $03(5.8)$ & \\
\hline \multicolumn{3}{|l|}{ Locality } & \multirow[t]{3}{*}{$\chi^{2}=19.883(p<0.001)$} \\
\hline Urban & $26(89.6)$ & $20(38.5)$ & \\
\hline Rural & $3(10.4)$ & $32(61.5)$ & \\
\hline \multicolumn{3}{|l|}{ Psychiatric diagnosis } & \multirow[t]{4}{*}{$\mathrm{FET}=1.44(p=0.49)$} \\
\hline Recurrent depressive disorder & $4(13.8)$ & $5(9.6)$ & \\
\hline Moderate depression & $21(72.4)$ & $43(82.7)$ & \\
\hline Dysthymia with moderate depression & $4(13.8)$ & $4(7.7)$ & \\
\hline
\end{tabular}

Abbreviations: FET, Fischer's exact test; HDRS, Hamilton's depression rating scale; IQR, interquartile range; SD, standard deviation. 
follow-up at 4 weeks. Again, 15 patients in the yoga group and 16 patients in the nonyoga group had completed 8 weeks of follow-up. No patients reported any adverse effects secondary to the yoga intervention. No worsening of mood symptoms was reported.

\section{Ease of Performing Asanas as Expressed by the Patients}

Twenty-eight patients (96.55\%) were able to learn all the asanas after the first session itself. Sixteen patients (55.2\%) expressed confidence for performing the asanas by themselves within a week of guided sessions. Nine patients (31.03\%) reported that they were able to remember and perform asanas by themselves on a daily basis at least for a month.

\section{Outcome details}

The patients receiving the adjunctive Kriya yoga intervention had significantly lower severity of depression (HDRS scores) at the end of 2, 4, and 8 weeks compared with the control group. The HDRS scores are reported in - Table 2.

The rates of remission (HDRS score $<7$ ) were significantly higher in the Kriya yoga group compared with the nonyoga group at the end of 2 and 4 weeks (-Table 3 ).

\section{Discussion}

The study demonstrates the feasibility of carrying out Kriya yoga intervention among patients with depression. Patients who received the adjunctive Kriya yoga intervention had significantly lower severity of depression at the end of 2, 4, and 8 weeks of intervention as per the HDRS. Similarly, patients who received the adjunctive Kriya yoga intervention had significantly greater remission at the end of 2 and 4 weeks of intervention (as per the HDRS score <7).
Our study finding is in line with several studies reporting the role of "Sudarshan Kriya Yoga" (SKY) in depression. SKY, which composes of cycling breathing with "Om" chanting, has some features in common with Kriya yoga. ${ }^{10}$ SKY was found to be beneficial in reducing depression, as well as enhancing the quality of life in those suffering with depression in various study groups. ${ }^{16,17}$ SKY was effective as an adjuvant treatment in patients with depression with poor response to antidepressants. ${ }^{18}$ The rates of remission noted in our study have been higher compared with those reported in the literature. $^{3}$ An important point to be noted in this context is multiple sessions with the therapist itself can be therapeutic as the remission rate in both the groups were high.

Yoga could be a mediator of early response to antidepressants. In our study, approximately $72 \%$ of patients showed remission by end of week 2 . This is in line with one more study from India where approximately $47 \%$ of patients with depression and low back ache achieved remission with 1 week of adjunctive yoga. ${ }^{19}$ Furthermore, depression was found to improve with greater duration of yoga intervention across studies. ${ }^{20}$ Hence, it would be interesting to assess dose-response relationship in future studies.

Though the feasibility of carrying out the intervention could be demonstrated in the index study, a discussion of challenges in carrying out the intervention is warranted. Logistical issues were one of the major problems faced by the cases while coming to the yoga center. They also had difficulty in taking out time from their schedules to visit the yoga center for 2 weeks.

To emphasize the strengths, it is probably the earliest study to show the feasibility of adjunctive Kriya yoga in depression. Furthermore, the confounding effect of antidepressant effect has been addressed as all the patients in both the groups were treated with escitalopram. It is interesting to note that in comparison to control group (30.7\%),

Table 2 Depression severity score (HDRS score) for cases and controls at follow-up

\begin{tabular}{|c|c|c|c|}
\hline Variable & Cases $(n=29)$ & Controls $(n=52)$ & $U$-value \\
\hline HDRS score & \multicolumn{2}{|l|}{ Mean (SD) } & \\
\hline 2 weeks (cases = 18; controls $=22$ ) & $5.28(4.15)$ & $12.36(5.44)$ & $U=56.5(p<0.001)$ \\
\hline 4 weeks $($ cases $=12 ;$ controls $=21$ ) & $2.17(2.12)$ & $8.58(3.90)$ & $U=21(p<0.001)$ \\
\hline 8 weeks $($ cases $=15 ;$ controls $=16)$ & $1.67(2.53)$ & $4.75(3.62)$ & $U=52(p=0.006)$ \\
\hline
\end{tabular}

Abbreviations: HDRS, Hamilton's depression rating scale; SD, standard deviation.

Table 3 Remission (as per HDRS score) for cases and controls at follow-up

\begin{tabular}{|c|c|c|c|}
\hline \multirow[t]{2}{*}{ Variable } & Cases $(n=29)$ & Controls $(n=52)$ & \multirow[b]{2}{*}{ Statistics } \\
\hline & \multicolumn{2}{|c|}{ Number of cases in remission $n(\%)$} & \\
\hline 2 weeks (cases = 18; controls = 22) & $13(72.22)$ & $3(13.63)$ & $\chi^{2}=14.16(p<0.001)$ \\
\hline 4 weeks $($ cases $=12 ;$ controls $=21$ ) & $12(100.00)$ & $5(23.80)$ & $\chi^{2}=17.75(p<0.001)$ \\
\hline 8 weeks $($ cases $=15 ;$ controls $=16$ ) & $14(93.33)$ & $13(81.25)$ & $\chi^{2} .=1.006(p=0.316)$ \\
\hline
\end{tabular}

Abbreviation: HDRS, Hamilton's depression rating scale. 
the follow-up rate at 8 weeks was significantly higher in the intervention group (51.7\%), which could denote the better acceptability of the Kriya yoga intervention. Also, the safety profile of the intervention has also been reported.

\section{Limitations and Future Directions}

However, it is imperative to interpret the study findings in the background of its limitations. First, the lower rates of follow-up is the biggest limitation. Around 70\% of controls had dropped out by the end of week 8 which could introduce bias. Second, the follow-up period was also shorter. Third, the study findings could be influenced by the differences in the sociodemographic profile. The majority of the cases belonged to the urban background and were students which could affect the generalizability of our study. Compliance to the intervention could be ensured only through phone calls. Some of the patients had logistic issues in commuting to the center because of which they dropped out. Finally, the study findings may not be generalizable as medical and psychiatric comorbidities were excluded.

To emphasize on implications, adjunctive Kriya yoga can help in improving patient outcomes by improving rates of clinical and functional remission. There is a need for further studies with rigorous study methodology such as RCT design, bigger sample size, and longer follow-up period to investigate feasible, acceptable, and effective intervention strategies for the patients with depression and other common mental disorders.

\section{Conclusion}

To conclude, the index study demonstrates the feasibility of yoga as an adjunctive treatment of depressive disorders in the Indian setting. Adjunctive Kriya yoga intervention resulted in significantly early response, lower severity of depression, higher rates of remission, and retention at the end of the intervention. However, further studies are needed to replicate our study findings to establish its efficacy.

\section{Authors' Contributions}

A.S.: conceptualized the project, participated in collection of data, analyzed the data, contributed to preparation of the manuscript, and edited and finalized the manuscript; P.P.K.: participated in collection of data, analyzed the data, contributed to preparation of the manuscript, and edited and finalized the manuscript; T.G.: conceptualized the project, participated in collection of data, and finalized the manuscript; N.N.: conceptualized the project, participated in collection of data, contributed to preparation of the manuscript, and edited and finalized the manuscript; A.C.: validation of the Kriya yoga intervention and execution of intervention program.

\section{Funding}

A.S. acknowledges the Indian Council of Medical Research, New Delhi, for providing the Short-Term Studentship (STS no.: 2018-01077).

\section{Conflict of Interest}

None declared.

\section{Acknowledgment}

The authors are grateful to Late Ms. Asha Chandani for her sincere contribution and logistic support for validation of Kriya yoga intervention and execution of intervention program at Indra Yog Sansthan.

\section{References}

1 World Health Organization. Depression in India, Let's Talk. Accessed December 28, 2020 at: https://ruralindiaonline.org/ library/resource/dep-ression-in-india-lets-talk/

2 Mendlewicz J. Towards achieving remission in the treatment of depression. Dialogues Clin Neurosci 2008;10(4):371-375

3 Cramer H, Anheyer D, Lauche R, Dobos G. A systematic review of yoga for major depressive disorder. J Affect Disord 2017;213:70-77

4 Sharma VK, Das S, Mondal S, Goswami U, Gandhi A. Effect of Sahaj Yoga on neuro-cognitive functions in patients suffering from major depression. Indian J Physiol Pharmacol 2006;50(4):375-383

5 Mehta P, Sharma M. Yoga as a complementary therapy for clinical depression. Complement Health Pract Rev 2010;15: 156-170

6 Khawaja NG, Duncanson K. Using the university student depression inventory to investigate the effect of demographic variables on Students' depression. J Psychol Counsell Schools 2008;18:195-209

7 Hofmann SG, Sawyer AT, Witt AA, Oh D. The effect of mindfulness-based therapy on anxiety and depression: a meta-analytic review. J Consult Clin Psychol 2010;78(2):169-183

8 Piet J, Hougaard E. The effect of mindfulness-based cognitive therapy for prevention of relapse in recurrent major depressive disorder: a systematic review and meta-analysis. Clin Psychol Rev 2011;31(6):1032-1040

9 Janakiramaiah N, Gangadhar BN, Naga Venkatesha Murthy PJ, Harish MG, Subbakrishna DK, Vedamurthachar A. Antidepressant efficacy of Sudarshan Kriya Yoga (SKY) in melancholia: a randomized comparison with electroconvulsive therapy (ECT) and imipramine. J Affect Disord 2000;57(1-3): 255-259

10 Sharma A, Barrett MS, Cucchiara AJ, Gooneratne NS, Thase ME. A breathing-based meditation intervention for patients with major depressive disorder following inadequate response to antidepressants: a randomized pilot study. J Clin Psychiatry 2017;78(1):e59-e63

11 Sarubin N, Nothdurfter C, Schüle C, et al. The influence of Hatha yoga as an add-on treatment in major depression on hypothalamic-pituitary-adrenal-axis activity: a randomized trial. J Psychiatr Res 2014;53:76-83

12 Bapat S. Psychophysiological analysis of Kriya Yoga as per Patanjala Yoga Sutra. Yoga Mimamsa 2016;48:18

13 Dave M, Bhogal MV. Effects of Pranavajapa (Om recitation) part one-an experimental study. Yoga Mimamsa 1988;27:19-28

14 Hamilton M. Development of a rating scale for primary depressive illness. Br J Soc Clin Psychol 1967;6(4):278-296

15 SPSS I. SPSS for Windows, (Version 15.0. 1.1). Chicago, IL: SPSS. 2007

16 Toschi-Dias E, Tobaldini E, Solbiati M, et al. Sudarshan Kriya Yoga improves cardiac autonomic control in patients with anxiety-depression disorders. J Affect Disord 2017;214:74-80

17 Shiju R, Thomas D, Al Arouj M, Sharma P, Tuomilehto J, Bennakhi A. Effect of Sudarshan Kriya Yoga on anxiety, depression, and 
quality of life in people with type 2 diabetes: a pilot study in Kuwait. Diabetes Metab Syndr 2019;13(3):1995-1999

18 Brown RP, Gerbarg PL. Sudarshan Kriya yogic breathing in the treatment of stress, anxiety, and depression: part I-neurophysiologic model. J Altern Complement Med 2005;11(1):189-201
19 Tekur P, Nagarathna R, Chametcha S, Hankey A, Nagendra HR. A comprehensive yoga programs improves pain, anxiety and depression in chronic low back pain patients more than exercise: an RCT. Complement Ther Med 2012;20(3):107-118

20 Bridges L, Sharma M. The efficacy of yoga as a form of treatment for depression. J Evid Based Complementary Altern Med 2017;22(4):1017-1028 